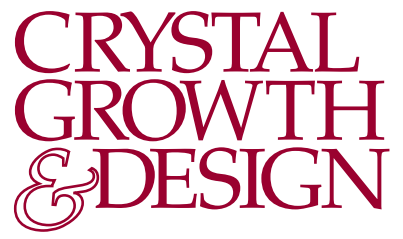

\title{
Crystal Growth of Lysozyme Controlled by Laser Trapping
}

\author{
Jing-Ru Tu, ${ }^{\dagger}$ Atsushi Miura, ${ }^{\dagger}$ Ken-ichi Yuyama, ${ }^{* \dagger}$ Hiroshi Masuhara, ${ }^{* \dagger}$ and Teruki Sugiyama ${ }^{*},+$ \\ ${ }^{\dagger}$ Department of Applied Chemistry and Institute of Molecular Science, National Chiao Tung University, Hsinchu 30010, Taiwan \\ ${ }^{\ddagger}$ Instrument Technology Research Center, National Applied Research Laboratories, Hsinchu 30076, Taiwan
}

\section{Supporting Information}

ABSTRACT: We demonstrate the growth of a tetragonal crystal of hen egg-white lysozyme (HEWL) in $\mathrm{D}_{2} \mathrm{O}$ buffer solution controlled by laser trapping with a focused continuous-wave $(\mathrm{CW})$ near-infrared (NIR) laser beam. The focal spot was located at $10 \mu \mathrm{m}$ away from the edge of the target crystal that was generated spontaneously, and the crystal growth was observed although the focal spot size was much smaller than the distance. The growth rate of (101) and $\{110\}$ faces of the tetragonal crystal was examined with various laser powers and polarizations. The rate observed under the irradiation was much different from those in spontaneous growth, namely, the growth rate of the $\{110\}$ face showed a large decrease or increase depending on the irradiation time. The dynamics and mechanism of this unusual crystal growth behavior is discussed from the viewpoint of a large stable domain formation of the HEWL liquidlike

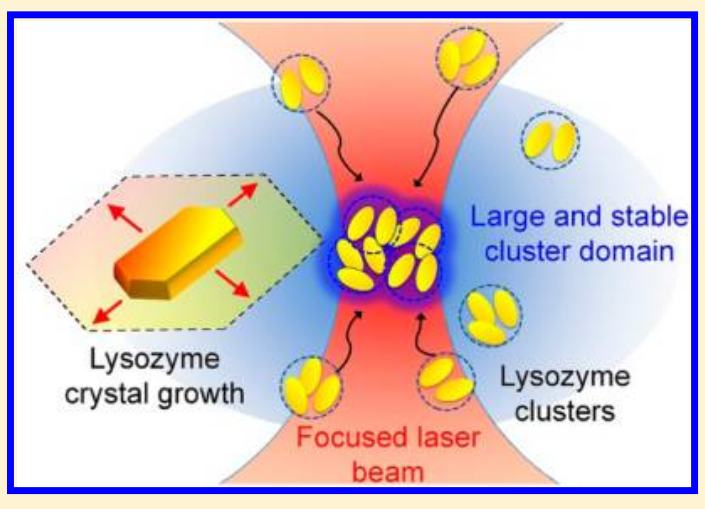
clusters through liquid nucleation and growth and by considering the anisotropy of the cluster domain.

$\mathrm{L}$ aser-induced nucleation and crystal growth have received much attention over the past two decades because of their wide applicability, polymorph selectivity, and spatial-temporal controllability. ${ }^{1-10}$ Most of the studies on laser-induced nucleation have been conducted using nanosecond ${ }^{1-5}$ and femtosecond ${ }^{6-8}$ pulsed lasers, and their mechanisms have been considered in terms of realignment of solutes ${ }^{1}$ and solvents ${ }^{3,4}$ under intense electromagnetic field such as optical Kerr effect and local concentration increase due to cavitation bubble generated by optical breakdown of solvent. ${ }^{7,8}$ In recent years, using an intense CW NIR laser beam, we have demonstrated laser trapping-induced crystallization of amino acids at the solution surface. $^{9-11}$ Laser trapping based on gradient force of a tightly focused laser beam can gather small targets such as nanoparticles, clusters, and polymers at the focal spot. ${ }^{12,13}$ The liquidlike clusters of amino acids in their solutions, in which solute and solvent are weakly linked with each other, ${ }^{14-16}$ are efficiently trapped at the focal spot, where the local concentration is nonlinearly increased with the irradiation time, ${ }^{9-11}$ and eventually their nucleation should be realized.

In 2009, we have succeeded in controlling crystal growth of glycine in saturated $\mathrm{D}_{2} \mathrm{O}$ solution utilizing this laser trapping technique. ${ }^{17}$ In that study, the CW NIR laser beam focusing at a glass/solution interface layer was set at about $20 \mu \mathrm{m}$ away from the edge of a crystal that had been generated spontaneously. Before the irradiation, the growth was completely stopped, while immediately after turning on the laser the crystal started growing toward the focal spot with an average growth rate of $10^{3} \mathrm{~nm} / \mathrm{s}$. It is noteworthy that the distance between the crystal edge and the focal spot is much larger than the focal spot size of approximately $1 \mu \mathrm{m}$ in diameter. We explained that this crystal growth is caused by formation of a large-volume, high-concentration region of glycine around the focal spot. The formation of such a region is strongly supported later by our finding that a millimeter scale lenslike dense liquid droplet of glycine is formed by laser trapping. ${ }^{18}$ This dense droplet is prepared by focusing the laser beam into a glass/solution interface of the glycine solution with a supersaturation value (SS) of 1.36 and eventually a large droplet of $5 \mathrm{~mm}$ in diameter with a SS of about 2.7 was formed. We suggested that the dense droplet is formed by the liquidliquid phase separation through liquid nucleation and following growth and the local high concentration region formed in a focal volume expands from the focal spot to its outside.

Many researchers have extensively developed crystallization techniques for proteins, sometimes utilizing lasers, ${ }^{2,6-8}$ because their crystals are necessary to determine three-dimensional protein conformation by X-ray structural analysis. Our first trial of laser trapping-induced nucleation of a protein was conducted for ferritin derivatives, where a two-dimensional disklike concentrated domain with a radius of a few tens of micrometers was formed but no crystal nucleation was realized. ${ }^{19}$ The achievement of the large domain formation under laser irradiation led us to think that protein crystal growth is controllable by laser trapping.

In this paper, we report laser trapping-controlled crystal growth of a tetragonal HEWL crystal. HEWL has been widely used as a model system for elucidating dynamics and mechanisms of nucleation and crystal growth of protein. ${ }^{20-23}$ The growth rate of two crystal faces with and without laser

Received: July 15, 2013

Revised: November 21, 2013

Published: November 22, 2013 


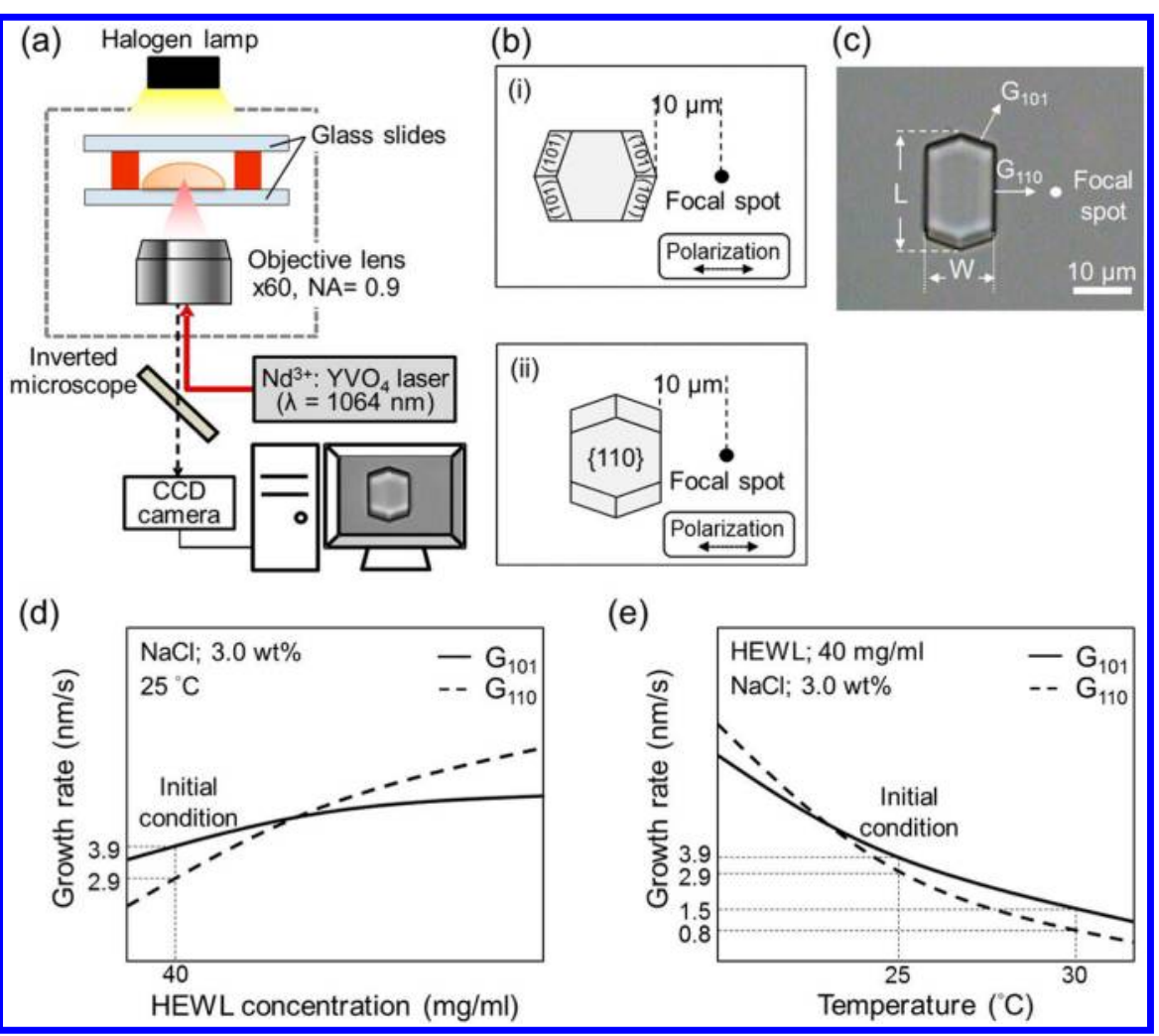

Figure 1. (a) Schematic illustration of the optical setup. (b) Focal position at $10 \mu \mathrm{m}$ from a crystal face of (i) (101) or (ii) \{110\}, which irradiation geometry is abbreviated as (101) or $\{110\}$ irradiation. The laser polarization direction is included. (c) The length $(L)$ and width $(W)$ defined for tetragonal HEWL crystal. Growth rate of $(101)$ and $\{110\}$ faces is calculated from length $(L)$ and width $(W)$ and illustrated as $G_{101}$ and $G_{110}$, respectively. See text for definitions of $G_{101}$ and $G_{110}$. (d and e) Schematic illustrations of HEWL crystal growth rate, depending on its concentration in solution and on temperature, respectively.

irradiation is investigated and compared. The dynamics and mechanism of the growth are considered in terms of the formation of a large stable domain of HEWL clusters through liquid nucleation and growth and anisotropy of the domain controlled by laser.

Commercially available HEWL (Wako, $>99.0 \%$ ) was used as a target protein without further purification. Since $\mathrm{H}_{2} \mathrm{O}$ has a higher absorption coefficient than $\mathrm{D}_{2} \mathrm{O}$ at $1064 \mathrm{~nm}$ of the trapping laser because of the overtones of the $\mathrm{OH}$ vibration, we prepared a buffer solution using $\mathrm{D}_{2} \mathrm{O}$ (Sigma-Aldrich) in order to suppress laser heating. Actually, the temperature elevation was estimated to be $22-24 \mathrm{~K} / \mathrm{W}$ in $\mathrm{H}_{2} \mathrm{O}$ and $2 \mathrm{~K} / \mathrm{W}$ in $\mathrm{D}_{2} \mathrm{O}$ by focusing a $1064 \mathrm{~nm}$ laser beam into each solution with a high numerical aperture $(\mathrm{NA})$ objective lens $(\mathrm{NA}=1.35, \times 100) \cdot{ }^{24} \mathrm{~A}$ HEWL stock solution $(80 \mathrm{mg} / \mathrm{mL})$ was prepared by dissolving HEWL powder into an acetate buffer solution (sodium acetate-acetic acid buffer in $\mathrm{D}_{2} \mathrm{O}, 100 \mathrm{mM}, \mathrm{pD}=5.1$ ). The $\mathrm{pD}$ value was corrected by adding 0.4 to the observed value of the $\mathrm{pH}$ meter. ${ }^{25}$ Equal volumes of the HEWL solution and $6.0 \%(\mathrm{w} / \mathrm{v}) \mathrm{NaCl}$ buffer solution were mixed just before the laser trapping experiments in order to avoid spontaneous nucleation. A small amount $(40 \mu \mathrm{L})$ of the mixed solution (HEWL, $40 \mathrm{mg} / \mathrm{mL} ; \mathrm{NaCl}, 3.0 \%(\mathrm{w} / \mathrm{v}) ; \mathrm{pD}=5.1$ ) was poured into a silicone chamber (Grace Bio-Labs, Inc., Press-To-Seal Silicone Isolator, $20 \mathrm{~mm}$ in diameter $\times 2.0 \mathrm{~mm}$ thickness), which has a glass substrate with a hydrophilic surface, and the sample was fully covered with another glass substrate to suppress solvent evaporation. At 40-60 min after the sample preparation, several spontaneously generated tetragonal crystals with a size of $10-30 \mu \mathrm{m}$ were observed in an inverted microscope. The mixed sample solution $(40 \mu \mathrm{L})$ was added again into the chamber in order to maintain the initial solution concentration. Thus, a thin film of solution was prepared, and its thickness was estimated to be $200-300 \mu \mathrm{m}$. The absorption coefficient of the solution at $1064 \mathrm{~nm}$ was measured using a spectrophotometer (HITACHI, U-4100) in order to estimate temperature elevation induced by laser irradiation.

Figure 1a shows a schematic illustration of the optical setup used in this work. A linearly polarized CW NIR laser beam of $1064 \mathrm{~nm}$ from a Nd ${ }^{3+}: \mathrm{YVO}_{4}$ laser (Coherent, Matrix 1064) was used as a trapping light source and focused near the glass/ solution interface (approximately $3 \mu \mathrm{m}$ above the glass surface) through an objective lens $(\mathrm{NA}=0.90, \times 60)$. The laser power throughout the objective lens was tuned at 0.6 or $1.1 \mathrm{~W}$ by adjusting the angle of a half-wave plate coupled with a polarizing beam splitter. The focal spot was set to $10 \mu \mathrm{m}$ from a crystal face of (101) or $\{110\}$ of a spontaneously formed tetragonal HEWL crystal (henceforth, the irradiation geometry is abbreviated as (101) or $\{110\}$ irradiation), as illustrated in Figure $1 \mathrm{~b}$. The crystal growth behavior with and without 30 min laser irradiation was monitored using a charge-coupled device (CCD) camera (Watec, WAT-231S2). The growth rate of (101) and $\{110\}$ faces (abbreviated to $\mathrm{G}_{101}$ and $\mathrm{G}_{110}$, respectively) was calculated from the $\mathrm{CCD}$ images captured every $5 \mathrm{~min}$. The entire experiment was conducted at room temperature $\left(25^{\circ} \mathrm{C}\right)$.

A tetragonal HEWL crystal consists of eight quadrilateral (101) and four hexagonal $\{110\}$ faces. Under our experimental conditions, spontaneous crystallization always provided the tetragonal crystal (Figure 1c). In most cases, the distances 


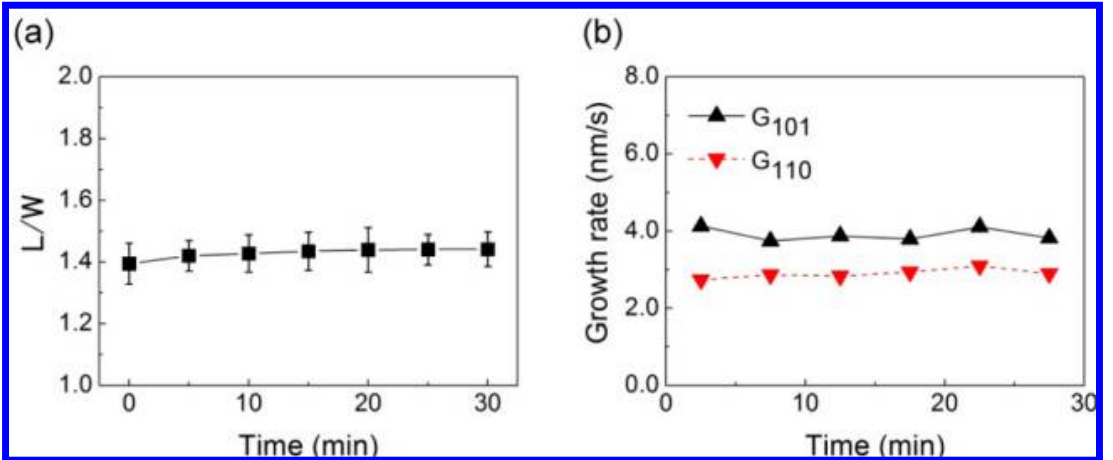

Figure 2. Temporal changes in the (a) shape parameter $(L / W)$ and $(b)$ growth rate for 30 min without laser irradiation at $25^{\circ} \mathrm{C}$. The plotted data were averaged over four samples, while the error bars in (a) indicate standard deviations at the respective irradiation times.

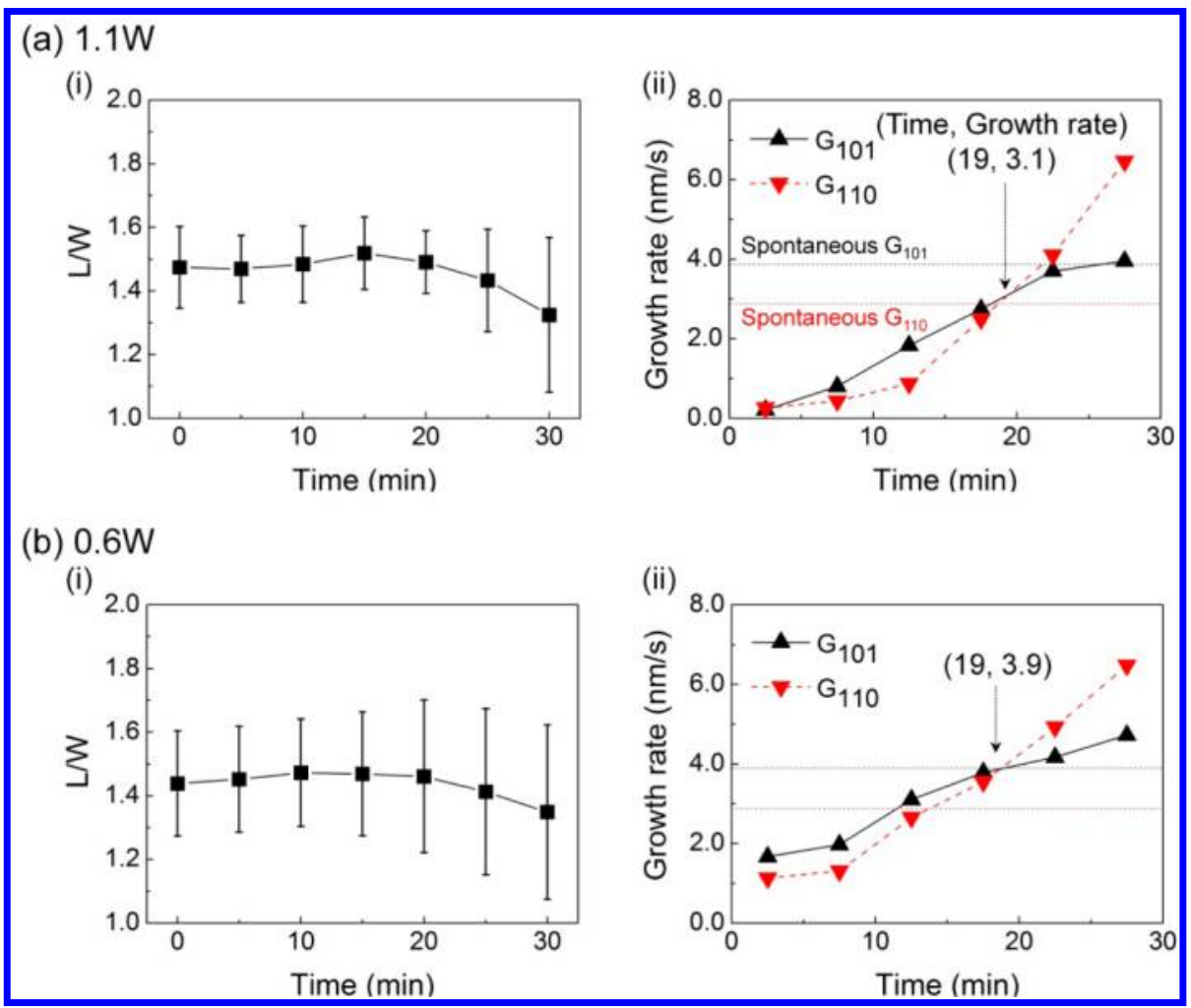

Figure 3. Temporal changes in the shape parameter $(L / W)$ and in the growth rate for 30 min under the (101) irradiation at (a) $1.1 \mathrm{~W}$ and (b) $0.6 \mathrm{~W}$ at $25{ }^{\circ} \mathrm{C}$. The plotted data were averaged over six samples; their standard deviation of the shape parameter was displayed, and the spontaneous growth rate is shown in panel (ii) as dotted lines.

between the generated crystals were far enough to set only one HEWL crystal in the observation area using the CCD camera $\left(80 \times 60 \mu \mathrm{m}^{2}\right)$, and one of them was selected as a target of further laser trapping experiments. The temporal changes of length $(L)$ and width $(W)$ shown in the figure were measured, and the growth rate of (101) and $\{110\}$ faces was estimated. Although our careful attention was paid to the asymmetric nature of crystal growth under laser irradiation, we never found the awkwardly shaped crystal during the irradiation. Therefore, the growth rate estimated here should be ascribed to the growth of all faces. The $\mathrm{G}_{101}$ and $\mathrm{G}_{110}$ are linked with $L$ and $W$ by the following equations as reported by Durbin and Feher. ${ }^{26}$

$$
G_{101}=0.45 \mathrm{~d} L / \mathrm{d} t, \quad G_{110}=0.5 \mathrm{dW} / \mathrm{d} t
$$

The $G_{101}$ and $G_{110}$ of a tetragonal crystal in aqueous buffer solution have been reported so far, and they strongly depend on many experimental parameters such as HEWL concen- tration, temperature, salt concentration, and $\mathrm{pH}$ of solution. ${ }^{26-28}$ In reference to those results, the changes in $G_{101}$ and $G_{110}$ against total HEWL concentration and temperature are schematically illustrated in Figure 1 (panels $\mathrm{d}$ and e), respectively. Our experiments were conducted in $\mathrm{D}_{2} \mathrm{O}$ buffer solution, so that we ourselves examined $G_{101}$ and $G_{110}$ in the $\mathrm{D}_{2} \mathrm{O}$ buffer solution under four different experimental conditions, and some of the results are shown in the figures (for experimental details, see the Supporting Information). Remarkably, $G_{101}$ and $G_{110}$ cross each other at a certain point, while both rates increase with the increase in HEWL concentration.

First, a HEWL crystal with a size of about $L=15 \mu \mathrm{m}$ was chosen as a target and the temporal changes of the shape parameter; $L / W$ and the growth rate were examined for four samples without laser irradiation. In Figure 2a, the averaged $L /$ $W$ gradually and slightly increased with time, indicating that the 
Table 1. Mean Value of $G_{101}$ and $G_{110}$ at $0-5$ and 25-30 min of the Irradiation at $25{ }^{\circ} \mathrm{C}$ Based on Panel (ii) of Figures 3 and 4 with their Standard Deviations

\begin{tabular}{|c|c|c|c|c|c|c|c|c|c|}
\hline focal position & $\begin{array}{l}\text { spontaneous crystal } \\
\text { growth }\end{array}$ & \multicolumn{4}{|c|}{ (101) } & \multicolumn{4}{|c|}{110} \\
\hline laser power $(\mathrm{W})$ & - & \multicolumn{2}{|c|}{1.1} & \multicolumn{2}{|c|}{0.6} & \multicolumn{2}{|c|}{1.1} & \multicolumn{2}{|c|}{0.6} \\
\hline $\begin{array}{l}\text { irradiation time } \\
\quad(\min )\end{array}$ & - & $0-5$ & $25-30$ & $0-5$ & $25-30$ & $0-5$ & $25-30$ & $0-5$ & $25-30$ \\
\hline$G_{110}(\mathrm{~nm} / \mathrm{s})$ & $2.9^{a}$ & $0.26 \pm 0.48$ & $6.5 \pm 4.6$ & $1.1 \pm 0.47$ & $6.5 \pm 5.0$ & $0.079 \pm 0.11$ & $3.7 \pm 4.4$ & $1.5 \pm 0.54$ & $4.3 \pm 1.5$ \\
\hline$G_{101}(\mathrm{~nm} / \mathrm{s})$ & $3.9^{a}$ & $0.21 \pm 0.45$ & $4.0 \pm 1.3$ & $1.7 \pm 0.53$ & $4.7 \pm 2.5$ & $0.77 \pm 0.50$ & $3.5 \pm 2.0$ & $1.7 \pm 0.62$ & $4.3 \pm 1.3$ \\
\hline
\end{tabular}

crystal growth accompanies a minute change in the morphology. Figure $2 \mathrm{~b}$ shows the temporal changes of averaged $G_{101}$ and $G_{110}$, and no appreciable temporal change in the growth rate was confirmed during the $30 \mathrm{~min}$ observation. Their mean rate for $30 \mathrm{~min}$ was 3.9 and $2.9 \mathrm{~nm} / \mathrm{s}$, respectively, which was also given in Figure 1 (panels $\mathrm{d}$ and $\mathrm{e}$ ), as a reference. The obtained $G_{110}$ was approximately 3 times larger than that in the aqueous buffer solution under similar solution conditions (about $1.0 \mathrm{~nm} / \mathrm{s}$ ). ${ }^{26,27}$ This result is possibly ascribed to the fact that the HEWL solubility in $\mathrm{H}_{2} \mathrm{O}$ is 1.3 times higher than that in $\mathrm{D}_{2} \mathrm{O} .{ }^{27}$ Most notably, $G_{110}$ never exceeded $G_{101}$ at this HEWL concentration.

Next, we present the crystal growth behavior under the (101) irradiation [Figure $1 \mathrm{~b}(\mathrm{i})]$. The direct observation was carried out for $30 \mathrm{~min}$, and after that the edge of the growing crystal reaches the focal spot. Figure 3 (panels a and b) shows the temporal changes of $L / W, G_{101}$, and $G_{110}$ under $1.1 \mathrm{~W}$ and 0.6 $\mathrm{W}$ irradiation, respectively. The average values were calculated for six samples under each condition, and the standard deviations (SD) of $L / W$ were given in the figure as an error bar (SD of $G_{101}$ and $G_{110}$ are shown in Table 1 ). At $1.1 \mathrm{~W}, L / W$ initially increased slightly until the $15 \mathrm{~min}$ irradiation and then decreased at 15-30 min. A similar temporal change of $L / W$ was observed also at $0.6 \mathrm{~W}$. The laser irradiation induced a time-dependent change in the crystal morphology, leading to a crystal shape much different from the spontaneous one.

This unusual growth behavior is considered by examining the temporal changes of $G_{101}$ and $G_{110}$, which are shown in panel (ii) of Figure 3 (panels a and b). At 1.1 and $0.6 \mathrm{~W}$, both $G_{101}$ and $G_{110}$ initially became much lower than the spontaneous ones and then gradually increased with the irradiation. In all cases, the growth rate showed monotonous increase with the irradiation. It might be possible that the monotonous increase in the growth rate is induced through solvent evaporation by laser heating due to light absorption. Indeed, our HEWL solution has absorption coefficient of $1.6 \mathrm{~m}^{-1}$ at $1064 \mathrm{~nm}$. With the assumption that temperature elevation at the focal spot is simply proportional to the absorption coefficient, it is calculated to be approximately $4 \mathrm{~K} / \mathrm{W}$; namely, the $1.1 \mathrm{~W}$ irradiation provides a temperature elevation of $4.4 \mathrm{~K}$. Because this heat diffuses from the focal spot to its outside, the actual temperature around the crystal does not exceed $30{ }^{\circ} \mathrm{C}$. We also confirmed that the $30 \mathrm{~min}$ laser irradiation always resulted in solvent evaporation of $3-5 \%$ volume, which was calculated by measuring the solution thickness before and after the irradiation. Importantly, the volume of solution evaporated was almost independent of the input laser power. This strongly indicates that vapor pressure in the chamber already reached the saturation value of the solution within the $30 \mathrm{~min}$ irradiation. In other words, the monotonous increase in the growth rate during the irradiation cannot be explained in view of the increase in HEWL concentration accompanying solvent evaporation.

It is notable that a crossover point of $G_{101}$ and $G_{110}$ was observed at a late stage of the irradiation. In accordance with Figure 1 (panels $\mathrm{d}$ and $\mathrm{e}$ ), the point appears at considerably high HEWL concentration and/or low temperature, where $G_{110}$ increases preferentially compared with $G_{101}$. It seems unlikely that laser irradiation induces temperature lowering; therefore, the appearance of the crossover point is thought to be due to the increase of HEWL concentration. In order to discuss how the solvent evaporation contributes to the growth rate, spontaneous crystal growth in a 5\%-solvent evaporated solution (HEWL; $42 \mathrm{mg} / \mathrm{mL}, \mathrm{NaCl} ; 3.15$ wt \%, pD = 5.1) was investigated at 25 and $30{ }^{\circ} \mathrm{C}$ (for details, see the Supporting Information). Under both conditions, spontaneous crystal growth proceeded at an almost constant growth rate as was the case in Figure $2 \mathrm{~b}$. At $30{ }^{\circ} \mathrm{C}, G_{101}$ and $G_{110}$ averaged for 30 min were estimated to be 2.4 and $1.6 \mathrm{~nm} / \mathrm{s}$, respectively. Even at $25{ }^{\circ} \mathrm{C}, G_{101}$ and $G_{110}$ showed comparable values (5.7 and 6.0 $\mathrm{nm} / \mathrm{s}$, respectively). The considerable increase in $\mathrm{G}_{110}$ was not observed under these possible conditions. Thus, this result clearly indicates that the large enhancement of $\mathrm{G}_{110}$ beyond the crossover point in panel (ii) of Figure 3 (panels a and b) cannot be induced only by condensation through solvent evaporation. It is reasonable that the large enhancement of $G_{110}$ is attributed to a much higher concentration domain around the target crystal. The domain around the crystal should be formed by laser trapping of the liquidlike clusters, ${ }^{16}$ in which lysozyme and solvents are weakly linked with each other through their mutual intermolecular interactions, similarly with the case of glycine. ${ }^{17}$

Another noteworthy result is the strong suppression of $G_{101}$ and $G_{110}$ at the early stage of the irradiation. For example, $G_{101}$ and $G_{110}$ in the initial $5 \mathrm{~min}$ irradiation at $1.1 \mathrm{~W}$ in Figure 3a (ii) were estimated to be 0.21 and $0.26 \mathrm{~nm} / \mathrm{sec}$, respectively, which are 10-20 times lower than those of the spontaneous growth. In order to exclude the possibility that the decrease in SS around the crystal by laser heating suppresses the crystal growth, we examined $G_{101}$ and $G_{110}$ of the spontaneous growth at $30{ }^{\circ} \mathrm{C}$ in the same solution concentration (for details, see the Supporting Information). Under this assumed condition, it is expected that the crystal growth is suppressed more compared to under the laser irradiation condition because the $4.4{ }^{\circ} \mathrm{C}$ temperature elevation is calculated for the focal volume. The $G_{101}$ and $G_{110}$ were 1.5 and $0.8 \mathrm{~nm} / \mathrm{sec}$, respectively, which were still much larger than those under the irradiation. One may suggest a possibility that anisotropic convection results in shape change of the crystal, but we have never observed awkwardly shaped crystal. Thus, we have to consider another mechanism of strong suppression of $G_{101}$ and $G_{110}$ at the early stage of the irradiation, not in terms of laser heating. 


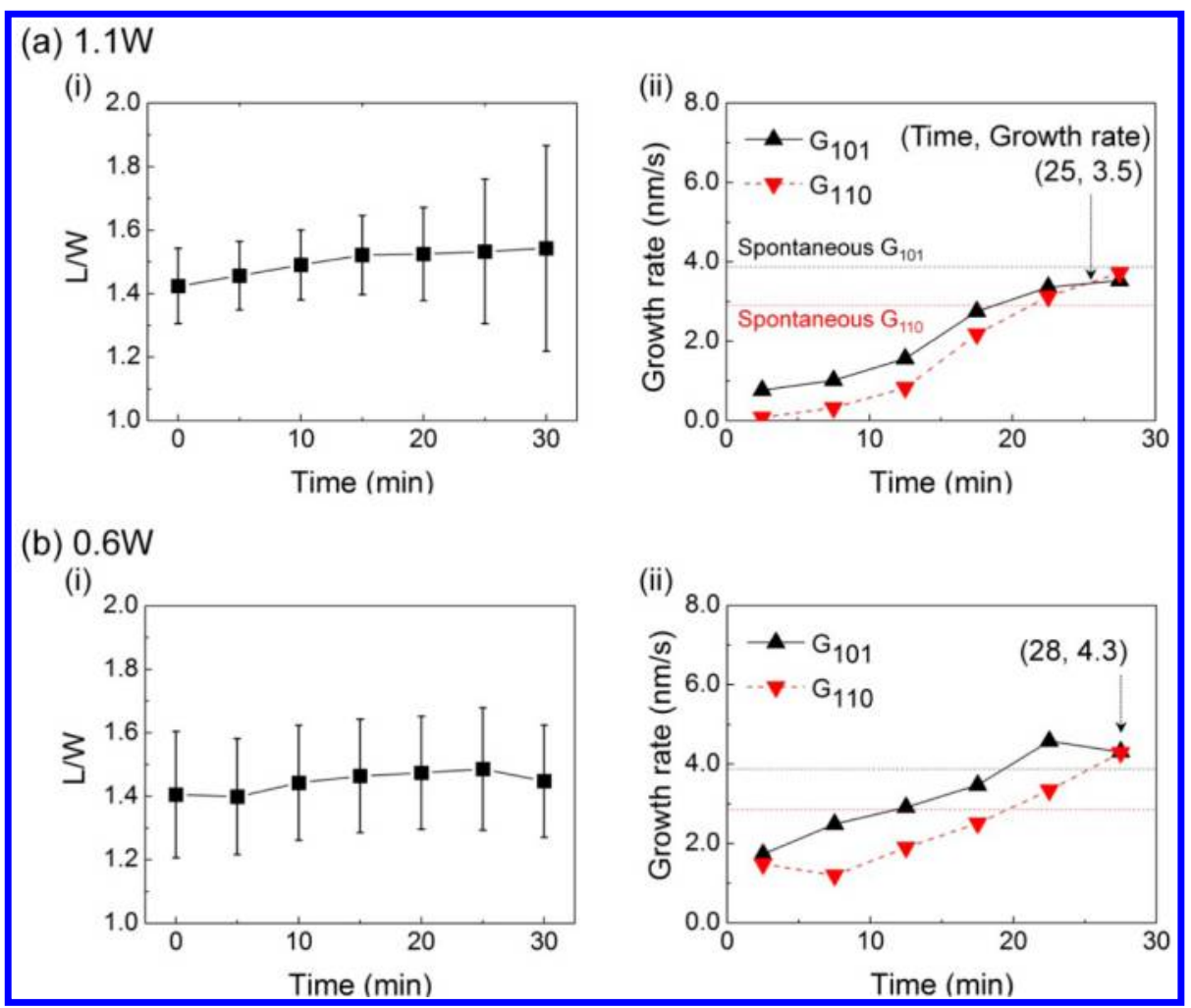

Figure 4. Temporal changes in the shape parameter $(L / W)$ and in the growth rate for 30 min under the $\{110\}$ irradiation at (a) $1.1 \mathrm{~W}$ and $(\mathrm{b}) 0.6$ $\mathrm{W}$ at $25{ }^{\circ} \mathrm{C}$. The plotted data were averaged over six samples; their standard deviation of the shape parameter was displayed, and the spontaneous growth rate is shown in the panel (ii) as dotted lines.

Before we discuss its details, we describe experimental results on growth kinetics controlled by the focusing position, which was carried out through the same experimental procedure as that under the $\{110\}$ irradiation [Figure $1 \mathrm{~b}$ (ii)]. Figure 4 (panels a and $\mathrm{b}$ ) show the temporal changes of $L / W, \mathrm{G}_{101}$, and $\mathrm{G}_{110}$ under $1.1 \mathrm{~W}$ and $0.6 \mathrm{~W}$ irradiation, respectively. At $1.1 \mathrm{~W}$, obtained $L / W$ increased continuously during the $30 \mathrm{~min}$ irradiation, unlike the temporal change under the (101) irradiation. A crossover point of $G_{101}$ and $G_{110}$ was observed, but it appeared later compared to the (101) irradiation. This delay is attributed to small enhancement of $G_{110}$ under the irradiation. No large enhancement of $G_{110}$ was observed at both laser intensities during the $\{110\}$ irradiation, while $G_{101}$ showed the temporal change similar to that under the (101) irradiation. Thus, we clearly confirmed the focal position dependence of the growth rate; namely, the growth behavior depends on the anisotropy of the domain formed by laser irradiation.

Table 1 shows the mean values with SD of the growth rate at 0-5 and 25-30 min of the irradiation for six samples based on panel (ii) of Figures 3 and 4. It is noteworthy that the initial 5 min irradiation causes strong suppression of the growth rate, which provides us much information for understanding the mechanism of the unusual crystal growth. We here propose three possibilities for the strong suppression. The first possibility is due to SS decrease by laser heating. Besides the above results of the spontaneous growth at $30^{\circ} \mathrm{C}$, this heating effect can also be excluded by our finding that the $G_{110}$ on the $\{110\}$ irradiation at $0.6 \mathrm{~W}$ was larger than that on the (101) irradiation. Under the $\{110\}$ irradiation, it is expected that the $G_{110}$ is suppressed more, due to the closer distance between the $\{110\}$ face and the focal spot compared to the (101) irradiation case. Thus, by considering all our results of this experiment, we can conclude that laser heating contributes little to this unique crystal growth behavior.

The second possibility is due to the decrease in the local concentration at the crystal surrounding area because many clusters are necessary for the formation of a high-concentration domain. In this case, it is suggested that the local concentration at a crystal face closer to the focal spot should be decreased more than that at the further face. Nevertheless, as described in the experimental part, we never found the awkwardly shaped crystal even at the early stage of the irradiation, which can deny this possibility. Therefore, the volume of the area is considerably smaller than that of the whole solution, and it is reasonable to consider that local concentration of the solution at the crystal edge is immediately balanced by the diffusion of the liquidlike clusters from the surroundings.

Thus, the strong suppression of $G_{101}$ and $G_{110}$ at the early stage of the irradiation is surely not due to temperature elevation by laser heating and local concentration decrease by laser trapping. Hence, we here propose that the strong suppression is due to rigidity and anisotropy of the domain formed by laser trapping. For the rigidity, the highconcentration domain by overcoming the energy barrier leading to nucleation of the dense domain is highly stable, so that their component clusters cannot be easily supplied to the target crystal. For the anisotropy, based on the obtained results on the crystal growth depending on the focal position, we consider that the liquidlike clusters are oriented anisotropically, according to the laser polarization direction; namely, the formed domain shows anisotropy. Mutual orientations between molecules at the crystal surface and liquidlike clusters determined by the anisotropy do not fit to crystal growth. 


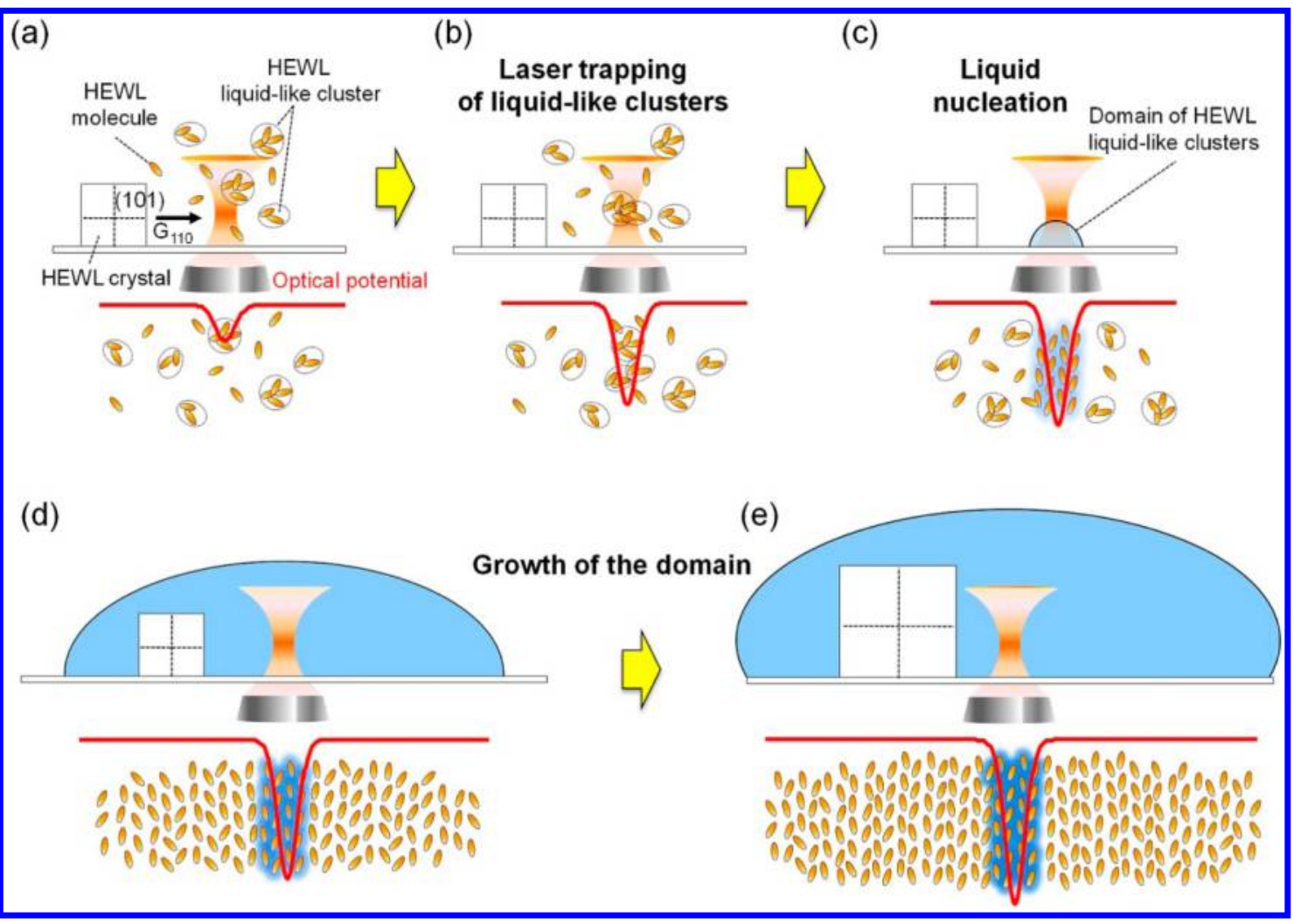

Figure 5. Schematic illustrations of prospective dynamics and mechanism of crystal growth of HEWL controlled by laser trapping.

At the present stage of the investigation, no clear microscopic picture of the domain is given, but we may refer to the results by Nadajara et al. and Sazaki et al. The former theoretically demonstrated that the crystal growth is realized by attaching HEWL cluster units such as tetramer, hexamer, and octamer to each (101) or $\{110\}$ crystal face, and their growth cluster units are attached to the crystal face. ${ }^{29,30}$ The different kinds of clusters are selectively attached to corresponding complementary sites on each crystal face. For example, the octamer are the predominant growth unit for the $\{110\}$ face. Sazaki and coworkers experimentally confirmed that the crystal growth of HEWL proceeds through adsorption of the cluster unit, not one molecule, to the crystal. ${ }^{31}$ On the basis of their results, we may interpret that the anisotropy and the rigidity of the domain which we proposed here determine the orientation of the cluster units and their attaching probability to the complementary sites of each crystal face, respectively.

Figure 5 summarizes the prospective dynamics and mechanism of this unusual crystal growth induced by laser trapping. We consider that the initial suppression and following increase of the growth rate are all caused by the formation of large stable domain of HEWL liquidlike clusters. First, the focused laser beam gathers and traps many liquidlike clusters of HEWL in the focal volume, while single HEWL molecule is too small to be trapped (Figure 5a). Such liquidlike HEWL clusters have been experimentally and theoretically investigated so far. $^{22,32-34}$ Once the clusters are trapped in the focus, their effective volume is increased, giving a stronger trapping force. Hence, optical potential becomes deeper nonlinearly with time and further efficient trapping of the clusters is induced (Figure $5 b)$. Their efficient trapping achieves very high concentration in the focal volume, and the energy barrier leading to nucleation of the dense domain is overcome. The nucleation triggers cluster packing with specific mutual orientation giving strong cluster interactions, which is called cluster domain here.

The small cluster domain prepared in the focal volume grows and expands from the focal spot to its outside due to mutual interactions between liquidlike clusters, convection flow, and mass transfer and fully covers the crystal for 5 min (Figure 5, panels $\mathrm{c}$ and $\mathrm{d}$ ). This timescale is consistent with our previous study on glycine solution, showing millimeter-scale dense liquid droplet formation by laser trapping. ${ }^{18}$ Thus, the growth rate depends just on the formed domain properties, not on its surrounding solution. The rigidity and anisotropy of the cluster domain decrease the growth rate in spite of its high concentration. The high concentration and anisotropy of the domain are formed only under laser irradiation and propagates to the outside (Figure 5e). This domain is stably kept as far as the trapping laser is introduced, and we here call this phenomena "laser trapping and pinning". Since the crystal growth rate increases as the crystal edge approaches the focal spot, the "laser trapping and pining" provides the highest concentration at the center of the focal spot, where the electric field of a Gaussian beam is strongest, and the pinning effect possibly becomes gradually weaker with the distance from the center of the focal spot. Furthermore, although no laser beam is directly illuminated outside of the focal spot, the concentration of the liquidlike clusters becomes high, and they are welloriented, all of which should be ascribed to mutual interactions between the clusters.

In conclusion, we have demonstrated that the crystal growth of HEWL in $\mathrm{D}_{2} \mathrm{O}$ buffer solution is well-controlled by laser trapping based on gradient force of a focused CW NIR laser beam. The temporal changes in crystal shape and growth rate of the (101) and $\{110\}$ faces were examined under various irradiation conditions. The growth behavior was much different 
from the spontaneous growth, in which the growth rate of the $\{110\}$ face was considerably decreased or increased, depending on the irradiation time. The dynamics and mechanism of the unusual crystal growth were considered in terms of laser trapping of liquidlike clusters, liquid nucleation, and following growth, formation of stable cluster domain, and rigidity and anisotropy of the domain. The rigidity and anisotropy of the domain are stably kept as far as the trapping laser is introduced, which phenomenon we call laser trapping and pining.

We believe that the success of the growth rate control of HEWL by laser trapping will also be extended to $3 \mathrm{D}$ precision control of crystal growth for other proteins and ensure the design of high-quality crystals large enough to be measured in single crystal X-ray crystallography. Further investigation on the cluster domain is necessary for deep understanding of the dynamics and mechanism of the crystal growth under laser trapping. We are now extending our studies of the cluster domain in view of 3D morphology, formation/growth dynamics, and internal cluster structure. The crystal growth experiments with combination of fluorescent dye labeledHEWL and spectroscopic/imaging techniques are being started in our group, which will provide us novel insights for this unusual growth behavior.

\section{ASSOCIATED CONTENT}

\section{S Supporting Information}

A series of experiments on laser trapping-induced crystal growth was carried out for a standard solution (HEWL; $40 \mathrm{mg}$ / $\mathrm{mL}, \mathrm{NaCl} ; 3.0$ wt \%, pD = 5.1). Since it was confirmed that $5 \%$ of the solvent evaporation was caused by laser irradiation, we examined how the solvent evaporation contributed to the growth rate of HEWL tetragonal crystal. The condensed solution (HEWL; $42 \mathrm{mg} / \mathrm{mL}, \mathrm{NaCl} ; 3.15$ wt \%, $\mathrm{pD}=5.1$ ) corresponding to $5 \%$ of the evaporation was prepared by mixing the HEWL solution $(80 \mathrm{mg} / \mathrm{mL}, 21 \mu \mathrm{L}), \mathrm{NaCl}$ buffer solution ( $8 \mathrm{wt} \%, 15.8 \mu \mathrm{L})$, and acetate buffer solution (100 $\mathrm{mM}, 3.2 \mu \mathrm{L}$ ). We investigated spontaneous growth rate for the standard and condensed samples at 25 and $30{ }^{\circ} \mathrm{C}$ because the temperature around the crystal during laser irradiation is considered to be from $25 \mathrm{C}^{\circ}$ to $30{ }^{\circ} \mathrm{C}$. A small amount $(40 \mu \mathrm{L})$ of the standard or condensed solution was poured into a glass substrate with a hydrophilic surface attached to a silicone chamber, and then the sample was fully covered with another glass substrate to avoid solvent evaporation. At 40-60 min after the sample preparation, several spontaneously generated tetragonal crystals with a size of $10-30 \mu \mathrm{m}$ were observed in an inverted microscope. A solution $(40 \mu \mathrm{L})$ identical to the initial one was added again into the chamber. We chose as the target crystal one of the spontaneously formed tetragonal crystals and then began the observation at $25{ }^{\circ} \mathrm{C}$. In the observation at $30{ }^{\circ} \mathrm{C}$, the sample was put on a thermo-plate (Tokai Hit, MATS-555RO) kept at $30^{\circ} \mathrm{C}$. The sample was aged for $10 \mathrm{~min}$ on the stage in order to homogenize the temperature, and then the observation of crystal growth behavior was started. The above experiment at $30{ }^{\circ} \mathrm{C}$ causes the solution evaporation, which induced a modest increase in the growth rate with time in $30 \mathrm{~min}$ of observation. Therefore, the values described in the main text are those observed on the initial $5 \mathrm{~min}$ observation. This material is available free of charge via the Internet at http://pubs.acs.org.

\section{AUTHOR INFORMATION}

\section{Corresponding Authors}

*E-mail: yuyama@nctu.edu.tw. Tel: +886 3 5712121. Fax: +88635723764.

*E-mail: masuhara@masuhara.jp. Tel: +886 3 5712121. Fax: +88635723764.

*E-mail: sugiyama@narlabs.org.tw. Tel: +886 3 5779911. Fax: +88635773947.

\section{Notes}

The authors declare no competing financial interest.

\section{ACKNOWLEDGMENTS}

The present work is partly supported by the National Science Council of Taiwan to T.S. (Grant NSC 102-2113-M-492-001MY2) and to H.M. (Grant NSC 100-2113-M-009-001), and the MOE-ATU Project (National Chiao Tung University) of the Ministry of Education, Taiwan, to H.M.

\section{REFERENCES}

(1) Garetz, B. A.; Aber, J. E.; Goddard, N. L.; Young, R. G.; Myerson, A. S. Phys. Rev. Lett. 1996, 77, 3475-3476.

(2) Lee, I. S.; Evans, J. M. B.; Erdemir, D.; Lee, A. Y.; Garetz, B. A.; Myerson, A. S. Cryst. Growth Des. 2008, 8, 4255-4261.

(3) Alexander, A. J.; Camp, P. J. Cryst. Growth Des. 2009, 9, 958-963.

(4) Duffus, C.; Camp, P. J.; Alexander, A. J. J. Am. Chem. Soc. 2009, 131, 11676-11677.

(5) Soare, A.; Dijkink, R.; Pascual, M. R.; Sun, C.; Cains, P. W.; Lohse, D.; Stankiewicz, A. I.; Kramer, H. J. M. Cryst. Growth Des. 2011, $11,2311-2316$

(6) Adachi, H.; Takano, K.; Hosokawa, Y.; Inoue, T.; Mori, Y.; Matsumura, H.; Yoshimura, M.; Tsunaka, Y.; Morikawa, M.; Kanaya, S.; Masuhara, H.; Kai, Y.; Sasaki, T. Jpn. J. Appl. Phys. 2003, 42, L798L800.

(7) Yoshikawa, H. Y.; Murai, R.; Sugiyama, S.; Sazaki, G.; Kitatani, T.; Takahashi, Y.; Adachi, H.; Matsumura, H.; Murakami, S.; Inoue, T.; Takano, K.; Mori, Y. J. Cryst. Growth 2009, 311, 956-959.

(8) Nakamura, K.; Sora, Y.; Yoshikawa, H. Y.; Hosokawa, Y.; Murai, R.; Adachi, H.; Mori, Y.; Sasaki, T.; Masuhara, H. Appl. Surf. Sci. 2007, 253, 6425-6429.

(9) Sugiyama, T.; Adachi, T.; Masuhara, H. Chem. Lett. 2007, 36, $1480-1481$.

(10) Yuyama, K.; Ishiguro, K.; Sugiyama, T.; Masuhara, H. Proc. SPIE 2012, 8458, 84582D-1-84582D-7.

(11) Sugiyama, T.; Yuyama, K.; Masuhara, H. Acc. Chem. Res. 2012, 45, 1946-1954.

(12) Ashkin, A. Proc. Natl. Acad. Sci. U.S.A. 1997, 94, 4853-4860.

(13) Neuman, K. C.; Block, S. M. Rev. Sci. Instrum. 2004, 75, 27872809.

(14) Hobbie, E. K. Phys. Rev. Lett. 1998, 81, 3996-3999.

(15) Stradner, A.; Sedgwick, H.; Cardinaux, F.; Poon, W. C. K.; Egelhaaf, S. U.; Schurtenberger, P. Nature 2004, 432, 492-495.

(16) Vekilov, P. G.; Pan, W.; Gliko, O.; Katsonis, P.; Galkin, O. Lect. Notes Phys. 2008, 752, 65-95.

(17) Sugiyama, T.; Adachi, T.; Masuhara, H. Chem. Lett. 2009, 38, 482-483.

(18) Yuyama, K.; Rungsimanon, T.; Sugiyama, T.; Masuhara, H. J. Phys. Chem. C 2012, 116, 6809-6816.

(19) Masuhara, H.; Sugiyama, T.; Rungsimanon, T.; Yuyama, K.; Miura, A.; Tu, J.-R. Pure Appl. Chem. 2011, 83, 869-883.

(20) Fiddis, R. W.; Longman, R. A.; Calvert, P. D. J. Chem. Soc., Faraday Trans. 1 1979, 75, 2753-2761.

(21) Sazaki, G.; Kurihara, K.; Nakada, T.; Miyashita, S.; Komatsu, H. J. Cryst. Growth 1996, 169, 355-360.

(22) Vekilov, P. G. Cryst. Growth Des. 2004, 4, 671-685.

(23) Vekilov, P. G. Cryst. Growth Des. 2010, 10, 5007-5019. 
(24) Ito, S.; Sugiyama, T.; Toitani, N.; Katayama, G.; Miyasaka, H. J. Phys. Chem. B 2007, 111, 2365-2371.

(25) Glasoe, P. K.; Long, F. A. J. Phys. Chem. 1960, 64, 188-190.

(26) Durbin, S. D.; Feher, G. J. Cryst. Growth 1986, 76, 583-592.

(27) Nadarajah, A.; Forsythe, E. L.; Pusey, M. L. J. Cryst. Growth 1995, 151, 163-172.

(28) Forsythe, E. L.; Nadarajah, A.; Pusey, M. L. Acta Crystallogr., Sect. D 1999, 55, 1005-1011.

(29) Nadarajah, A.; Li, M.; Pusey, M. L. Acta Crystallogr., Sect. D: Biol. Crystallogr. 1997, 53, 524-534.

(30) Li, M.; Nadarajah, A.; Pusey, M. L. Acta Crystallogr., Sect. D: Biol. Crystallogr. 1999, 55, 1012-1022.

(31) Matsui, T.; Sazaki, G.; Hondoh, H.; Matsuura, Y.; Nakada, T.; Nakajima, K. J. Cryst. Growth 2006, 293, 415-422.

(32) Streets, A. M.; Quake, S. R. Phys. Rev. Lett. 2010, 104, 1781021-178102-4.

(33) ten Wolde, P. R.; Frenkel, D. Science 1997, 277, 1975-1978.

(34) Erdemir, D.; Lee, A. Y.; Myerson, A. S. Acc. Chem. Res. 2009, 42, 621-629. 